\title{
Symmetry of Dislocation Images in Transmission Electron Microscopy
}

\author{
BY F. W. SCHAPINK \\ Laboratorium voor Metaalkunde, Technische Hogeschool, Delft, The Netherlands
}

(Received 16 May 1974)

\begin{abstract}
The application of the reciprocity theorem in the field of electron diffraction has led to a number of useful relations between intensities of various diffracted electron beams [Pogany, A. P. \& Turner, P. S. (1968). Acta Cryst. A24, 103]. Apart from perfect crystals, the theorem can also be applied to images of individual lattice defects, e.g. planar faults and dislocations [Howie, A. (1972). Proc. Fifth European Congress Electron Microscopy, Manchester, p. 408; Schapink, F. W. (1973) Phys. Stat. Sol. (b), 56, K61]. The symmetry relations for dislocation images, both in bright field and dark field, are investigated in some detail. They may be divided into two groups: (i) Symmetry due to application of the reciprocity theorem, followed by a symmetry operation of the crystal associated with the diffraction vector (mirror inversion or central inversion). The relations found do not depend on the specific properties of the dislocation displacement field. (ii) Special relations due to symmetry properties of the displacement field, for particular dislocation geometries. The resulting symmetry depends on the degree of elastic anisotropy of the crystal.
\end{abstract}

J. Appl. Cryst. (1975). 8, 220

\section{On Some Integral Aspects of the Dynamical Theory of Electron Diffraction}

\author{
By A. LANNES \\ Laboratoire d'Optique Electronique CNRS, 29, rue Jeanne Marvig, B.P. 4007, 31055 Toulouse Cedex, France
}

(Received 16 May 1974)

As is well known, the dynamical theory of electron diffraction can be developed by using the basic statement of Bethe, H. [Ann. Phys. Lpz. (1928). 87, 55] or integral treatments such as those of Fujiwara, K. [J. Phys. Soc. Japan, (1959), 14, 1513], Kambe, K. [Z. Naturforsch. (1967), 22a, 422], Gjønnes, J. [Z. Naturforsch, (1972), 27a, 3] and Lannes, A. [Phys. Stat. Sol. (b), (1973), 56, 513]. In a general way, the integral methods of approach seem preferable. Effectively, they are more general and more correct while at the same time facilitating mathematical and physical analysis of the diffusion problem. Some particular aspects of integral formalisms are of great interest in electron microscopy. In particular, integral methods allow us to introduce in a natural way the basic approximations of the dynamical theory of electron diffraction [Howie, A. \& Basinski, Z. S. (1968). Phil. Mag. 17, 1039; Lannes (1973); Gjønnes (1972)] (Forward diffusion approximation - Second, first and zeroth order approximation - Kinematical approximation). As an example, starting from the basic expressions of forward and backward diffusion [cf. relation (23) of Lannes (1973)]

where

$$
\hat{\varphi}_{g}^{ \pm} \simeq\left[\hat{\beta}_{g}^{ \pm} \otimes \dot{\delta}_{0}(z)+i \pi \hat{t}_{g}\right]_{z}^{*}\left[ \pm y(z) \frac{\exp \left(2 i \pi k_{g}^{ \pm} z\right)}{q_{g}}\right]
$$

$$
\begin{aligned}
& \hat{\beta}_{g}^{ \pm}=\frac{\hat{\varphi}_{g}^{\prime}(\chi, 0)}{4 i \pi}-\frac{1}{2} k_{g}^{\mp} \hat{\varphi}_{g}(\chi, 0), \quad t_{g}=\alpha \sum_{k} v_{g-h} \varphi_{h} \\
& k_{g}^{ \pm}=-k_{g} \pm q_{g}, \quad q_{g}^{2}=k_{g}^{2}+\varepsilon_{g}^{2}-w_{g}^{2}(\chi), \quad \varepsilon_{g}^{2}=K_{0}^{2}-K_{g}^{2},
\end{aligned}
$$

and neglecting the term of backward diffusion as well as $w_{g}^{z}$, one obtains the integral expressions relative to the column approximation (zeroth order approximation)

or alternatively

$$
\left.\varphi_{g}=\left[\varphi_{g}^{0} \otimes \delta_{0}+i \frac{\pi}{k_{g}} t_{g}\right] \stackrel{*}{*} Q_{g} \quad \text { where } \quad Q_{g}=y(z) \exp 2 i \pi t_{g}^{+} z\right)
$$

$$
\varphi_{g}(\mathbf{r}, z)=\left[\exp \left(2 i \pi \tau_{g}^{+} z\right)\right]\left[y(z) \varphi_{g}^{0}+i-\frac{\pi}{k_{g}} \cdot \int_{0}^{l} t_{g}(\mathbf{r}, \zeta) \exp \left(-2 i \pi \tau_{g}^{+} \zeta\right) \mathrm{d} \zeta\right]
$$

